\title{
ENHANCED ELECTRON ATTACHMENT TO HIGHLY-EXCITED MOLECULES AND ITS APPLICATIONS IN PULSED PLASMAS
}

\author{
L. A. Pinnaduwage ${ }^{1,2}$, W. X. Ding', D. L. McCorkle ${ }^{1,2}$, C. Y. Ma' \\ 'Oak Ridge National Laboratory, Oak Ridge, TN 3783 1, USA \\ ${ }^{2}$ The University of Tennessee, Knoxville, TN 37996, USA
}

\section{Abstract}

Studies conducted over the past several years have shown that electron attachment to highly-excited states of molecules have extremely large cross sections. We will discuss the implications of this for pulsed discharges used for $\mathrm{H}^{\cdot}$ generation, material processing, and plasma remediation.

\section{INTRODUCTION}

By highly-excited states we specifically mean highRydberg (HR) states which have electronic binding energies of a fraction of an electron volt (eV). Rydberg states of atoms and molecules have some unique characteristics including long lifetimes and large cross sections for various collision processes [ 1]. In a molecule, energy can be stored in the vibrational/rotational degrees of freedom in addition to its electronic energy. Therefore, a neutral molecule may have a total internal energy exceeding its lowest ionization potential (IP); each vibrational/rotational mode will have a Rydberg series converging to its own IP, and these can be called "core-excited" Rydberg states. Recent experimental studies [2-4] have shown that molecules excited to a few $\mathrm{eV}$ above their lowest IP can have microsecod lifetimes in their core-excited Rydberg states. Therefore, our following discussion applies to core-excited HR states as well as regular HR states.

In this paper we wish to point out that the HR states are likely to play a significant role in pulsed plasmas. The collision cross sections for HR states can be many orders of magnitude larger compared to those for ground state or lowlying excited states [1]; while the cross sections associated with ground state molecules are of the order of $10^{-16} \mathrm{~cm}^{2}$, cross sections for HR states can be $10^{-10} \mathrm{~cm}^{2}$ or even higher. Therefore, even if a fraction of molecules in a given plasma are excited to HR states, their collision processes may influence the properties of the plasma.

Our electron attachment studies on laser-excited HR states of molecules have shown that they have characteristically large cross sections for dissociative electron attachment; see [5] and references therein. Recently we have initiated studies on electron attachment to HR states in gas discharges: (i) we observed electron attachment to HR states of a number of molecules populated via excitation transfer from the metastable states of inert gas atoms that are efficiently populated in glow discharges [6, 7], and (ii) we observed $\mathrm{O}^{-}$formation in a pulsed $\mathrm{O}_{2}$ discharge, which we have shown to be due to enhanced electron attachment to HR states of $\mathrm{O}_{2}$ [8]. In pulsed discharges the conditions for electron attachment to HR states are optimized in the discharge "off" period, i.e., the afterglow: (i) the HR states of the molecules survive well into the post discharge due to their characteristically-long lifetimes, and (ii) the discharge electrons are rapidly cooled in the post discharge thus producing low-energy electrons that are conducive to electron attachment.

We will point out the possible implications of electron attachment to highly-excited states of molecules in three pulsed power technologies: (i) Generation of $\mathrm{H}^{-}$ions using $\mathrm{H}_{2}$ discharges is actively being pursued due to the need for intense $\mathrm{H}^{-}$ion beams for magnetic fusion energy research. It has recently been shown that higher average $\mathrm{H}^{-}$beam currents can be extracted in a pulsed $\mathrm{H}_{2}$ discharge source [9-14]; (ii) Recent studies show that power modulation enhances deposition/etching while reducing undesirable particulate formation in plasma processing discharges used in the processing of semiconductors [15-23]; (iii) Our recent studies [24] show that the energy efficiency in plasma remediation of volatile organic compounds in rare gas mixtures is enhanced in pulsed glow discharges. The above observations are likely to be related to enhanced dissociative electron attachment to HR states as described below.

\section{PULSED HYDROGEN DISCHARGES}

Generation of $\mathrm{H}$ ions using $\mathrm{H}_{2}$ discharges is actively being pursued due to the need for intense $\mathrm{H}$ ion beams for particle accelerators and for magnetic fusion energy research. Enhanced electron attachment to vibrationallyexcited states of the ground electronic state of $\mathrm{H}_{2}$ was reported $[25,26]$ at about the same time the efficient $\mathrm{H}$ formation in $\mathrm{H}_{2}$ discharges was reported [27]. Ever since that time, an exhaustive experimental and theoretical effort has been devoted to understand the experimental observations in the $\mathrm{H}_{2}$ discharge sources in terms of this electron attachment process (see, for example, [28]). However, recent studies [29-31] show that the experimentally measured $\mathrm{H}^{-}$number densities can not be completely accounted for by electron attachment to highvibrational (HV) states. Based on our observations on 
electron attachment to laser-excited $\mathrm{H}_{2}$ [32], we recently pointed out [33] that highly-excited electronic states of $\mathrm{H}_{2}$, which are high-Rydberg (HR) states, populated in $\mathrm{H}_{2}$ discharges may account for the difference, also see $[34,35]$. Evidence to support [36-38] and oppose [39] this proposal have been presented.

It has been recently shown $[9,10]$ that higher average $\mathrm{H}$ beam currents can be extracted by using pulsed discharges of $\mathrm{H}_{2}$. Subsequent studies [ $11-14$ ] have confirmed these observations and have presented further experimental observations designed to understand the reasons for this enhancement. Model calculations based on electron

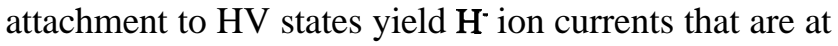
least 4 times smaller than the experimentally-measured values [40].

We believe that the enhanced production of $\mathrm{H}^{-}$signal in [12], as well as in the other pulsed $\mathrm{H}_{2}$ discharge experiments is due to, (i) the reduction in electron impact ionization of HR states, and (ii) the increased availability of slow electrons, in the post discharge. Further experiments are progress in our laboratory to test this hypothesis.

\section{PULSED MATERIAL PROCESSING DISCHARGES}

Most plasma processing discharges use gas mixtures consisting of a "reactive gas" and an inert gas. Thus in addition to the population of excited states of the reactive gas by electron impact, they can also be populated by excitation transfer from metastable states of the inert gas as we have illustrated in recent experiments $[6,7]$.

By monitoring the electron decay time in the afterglow of a silane/helium pulsed discharge, Fleddermamr et al. [41] have shown that the dominant electron loss mechanism in the afterglow was dissociative electron attachment (nondissociative electron attachment is not viable at the low pressures used in these experiments.) They estimated [4 1] a lower bound value of $\sim 2.6 \times 10^{-10} \mathrm{~cm}^{3} \mathrm{~s}^{-1}$ for the electron attachment rate constant involved; yet this is an order of magnitude higher than the electron attachment rate constant corresponding to the measured electron attachment cross sections measured for the ground state of silane [42]. Fleddermann et al. [4 1] proposed that this enhanced electron attachment in the afterglow was' to $\mathbf{S i H}_{3}$ or $\mathbf{S i H}_{2}$ radicals that were produced during the discharge. However, this possibility can be ruled out as shown below.

Negative ions from a pulsed silane discharge have been mass identified recently by Howling et al. [43,44]. They reported that monosilicon negative ions are initially produced by electron attachment and subsequently these polymerize to produce polysilicon negative ions; the dominant monosilicon negative ion produced by electron attachment is $\mathrm{SiH}_{3}{ }^{-}[43,44]$. Since it is not possible to produce the $\mathrm{SiH}_{3}$-ion via dissociative electron attachment to $\mathrm{SiH}_{3}$ (non-dissociaitve electron attachment is weak under low-pressure conditions) or $\mathrm{SiH}_{2}$ radicals, it is clear that the enhanced production of $\mathrm{SiH}_{3}{ }^{-}$ion in the afterglow must come from the parent $\mathrm{SiH}_{4}$ molecule. Since the ground state electron attachment for silane is more than an order of magnitude smaller than the experimentally measured value in the afterglow of a pulsed discharge [41], the only other explanation is dissociative electron attachment to highlyexcited states of silane. This is also consistent with our recent observation [5] of large electron attachment rate constants of the order of $10^{-7} \mathrm{~cm}^{3} \mathrm{~s}^{-1}$ for highly-excited states of silane produced via laser irradiation.

In plasma processing of semiconductor materials, it has been shown that pulsed discharges yield faster deposition with improved film quality [16-18, 20, 23, 45] and faster etching with reduced notching [15, $21,46-49]$.

This enhanced performance could be due, partly, to the enhanced dissociative electron attachment that occurs at the falling edge of a pulse. Dissociative electron attachment leads to the formation of radicals and fragment negative ions (which are charged radicals that can contribute to anisotropic etching as well as deposition). In a cw discharge, the negatively-charged particles are trapped in the discharge due to the sheath potentials and are allowed to grow into particulates. (Thus, the negative ions are also prevented from contributing to deposition/etching). Once they become micrometer-size particulates, the viscous drag force becomes large enough to overcome the electrostatic forces and they can reach the substrate. Therefore, the cw operation of the discharge is detrimental in two ways: (a) it prevents the negative ions from reaching the substrate and thus possibly contributing to the etching/deposition (furthermore, in etching applications the negative ions that reach the substrate can neutralize the positive ions, and thus reduce "notching"), and (b) it traps the negative ions in the discharge, thus allowing them to grow to pm-size particulates.

By time-modulating the discharge, the negative ions populated during the "on-time" are allowed to reach the substrate during the "off-time" (when the sheath fields are collapsed) and thus deposition/etching is enhanced while, at the same time, particulate growth is prevented. In addition, the negative-ion formation itself may be enhanced at the time the discharge is turned off and thus the flux of negative ions to the substrate is further enhanced during the discharge off period.

\section{PULSED PLASMA REMEDIATION}

We recently studied the destruction of benzene in a benzene/Ar mixture subjected to a pulsed glow discharge [24]. The destruction efficiency was observed to be much improved compared to a DC glow discharge [50]. Diagnostic spectroscopic measurements indicated that excitation transfer from the metastable states of Ar to benzene in the afterglow of the discharge was primarily 
responsible for the destruction of benzene.

Furthermore, in these experiments [24], it was observed that no detectable gaseous byproducts (other than Ar) appeared at the reactor output. Instead we observed a crystalized residue (dust) on the walls of the reactor and on the electrodes. These dust particulates appear to be similar to the diamond particulates observed in benzene discharges used for material processing; see, [51] and references therein. Since negative ions have been suggested to be the precursors for such particulate formation [52], it is quite possible that electron attachment to HR states of benzene contributed significantly to the dissociation of benzene in our experiments (electron attachment to ground state benzene is extremely weak).

The destruction efficiency was observed to enhance with decreasing pulse width and increasing ambient (Ar gas) pressure [24]. For a given energy density delivered to the plasma at a fixed frequncy, narrow pulse width means high discharge voltage and current; the high energy electrons thus enhanced the formation of metastable atoms. Narrow pulse width also limited further heating of the electrons. By terminating the discharge before the electrons were further heated, one can maintain the metastable atom density without further energy input. In the off-cycle, these metastable atoms transferred their energy to the benzene molecules. The core-excited HR states of benzene thus produced efficiently attached the electrons that were cooled down in the post discharge period (electron attachment to HR states is optimized at low electron energies). Therefore, narrow pulse width led to a better destruction efficiency.

The enhancement of the destruction efficiency with increasing pulse width can also be understood in terms of faster cooling of the electrons in the afterglow. Further studies are in progress in our laboratory in order to firmly establish the destruction mechanisms involved.

\section{SUMMARY}

We have discussed the recent observations on enhanced electron attachment to highly-Rydberg states of molecules and its implications for various pulsed discharges, including those used in $\mathrm{H}^{-}$generation, plasma processing, and plasma remediation.

*Research sponsored by the DOE-EMSP Program and the National Science Foundation. ORNL is managed by the Lockheed Martin Energy Research Corp. for the Department of Energy under contract number DE-AC0596OR22464.

\section{REFERENCES}

1. R. F. Stebbings and F. B. Dunning, eds. Rydberg States of Atoms and Molecules. 1983, Cambridge Universitv Press: Cambridge.
2. W. G. Scherzer, H. L. Selzle, E. W. Schlag, and R. D. Levine, Phys. Rev. Lett., 72, 1435 (1994).

3. H. Matsui, J. M. Behm, and E. R. Grant, Journal of Physical Chemistry A, 101, 6717 (1997).

4. L. A. Pinnaduwage and Y. Zhu, Chem. Phys. Lett., 277, 147 (1997).

5. L. A. Pinnaduwage and P. G. Datskos, Journal of Applied Physics, 81, 7715 (1997).

6. L. A. Pinnaduwage, W. X. Ding, and D. M. McCorkle, Appl. Phys. Lett., 71, 3634 (1997).

7. W. X. Ding, D. M. McCorkle, and L. A. Pinnaduwage, Journal of Applied Physics, 84, 3051 (1998).

8. W. X. Ding, L. A. Pinnaduwage, C. Tav, and D. M. McCorkle, Plasma Sources Sci. Technol., 8, 384 (1999).

9. M. B. Hopkins and K. N. Mellon, Phys. Rev. Lett., 67,449 (1991).

10. M. B. Hopkins, M. Bacal, and K. N. Mellon, Journal of Applied Physics, 70, 2009 (1991).

11. R. M. A. Heeren, K. N. Mellon, M. B. Hopkins, D. Ciric, and A. W. Kleyn, Europhys. Lett. , 17, 503 (1992).

12. K. N. Mellon, B. P. Coonan, and M. B. Hopkins, J. Phys. D, 27, 2480 (1994).

13. K. N. Mellon, B. P. Coonan, and M. B. Hopkins, Plasma Sources Sci. Technol., 4,591 (1995).

14. T. Mosbach, H. M. Katsch, and H. F. Dobele, Plasma Sources Sci. Technol., 7, 75 (1998).

15. R. W. Boswell and D. Henry, Appl. Phys. Lett., 47, 1095 (1985).

16. L. J. Gverzet and J. T. Verdeyen, Appl. Phys. Lett., 48,695 (1986).

17. Y. Watanabe, M. Shiratani, Y. Kubo, I. Ogawa, and S. Ogi, Appl. Phys. Lett., 53, 1263 (1988).

18. J. T. Verdeyen, J. Beberman, and L. Gverzet, J. Vac. Sci. Technol. A, 8, 1851 (1990).

19. A. Lloret, E. Bertran, J. L. Andujar, A. Canillas, and J. L. Morenza, Journal of Applied Physics, 69,632 (1991).

20. J. L. Andujar, E. Bertran, A. Canillas, J. Campmany, J. Serra, and C. Roch, Journal of Applied Physics, 71, 1546 (1992).

21. S. Samukawa, Appl. Phys. Lett., 64, 3398 (1994).

22. S. Samukawa and T. Mieno, Plasma Sources Sci. Technol., 5, 132 (1996).

23. A. Hatta, H. Suzuki, K. Kadota, H. Makita, T. Ito, and A. Hiraki, Plasma Sources Sci. Technol., 5, 235 (1996).

24. D. M. McCorkle, W. X. Ding, C. Y. Ma, and L. A. Pinnaduwage, Journal of Applied Physics, submitted (1999).

25. M. Allen and S. F. Wong, Physical Review Letters, 41, 1791 (1978).

26. J. M. Wadehra and J. N. Bardsley, Phys. Rev. 
Lett., 41, 1795 (1978).

27. M. Bacal, and G. W. Hamilton, Phys. Rev. Lett., 42, 1538 (1979).

28. W. G. Graham, Plasma Sources Sci. Technol., 4, 281 (1995).

29. P. J. Eenshuistra, M. Gochitashvilli, R. Becker, A. W.Kleyn, and H. J.Hopman, J. Appl. Phys., 67, 85 (1990).

30. P. Berlemont, D.-A. Skinner, and M. Bacal, Rev. Sci. Instrum., 64, 2721 (1993).

31. A. T. Young, P. Chen, K. N. Leung, and G. C. Stutzin, Rev. Sci. Instrum., 65, 1416 (1994).

32. L. A. Pinnaduwage and L. G. Christophorou, Phys. Rev. Lett., 70,754 (1993).

33. L. A. Pinnaduwage and L. G. Christophorou, Journal of Applied Physics, 76, 46 (1994).

34. P. G. Datskos, L. A. Pinnaduwage, and J. F. Kielkopf, Phys. Rev. A, 55, 4131 (1997).

35. L. A. Pinnaduwage, W. X. Ding, D. M. McCorkle, S. H. Lin, A. M. Mebel, and A. Garscadden, Journal of Applied Physics, 85, 7064 (1999).

36. A. Garscadden and R. Nagpal, Plasma Sources Sci. Technol., 4,268 (1995).

37. K. Hassouni, A. Gicquel, and M. Capitelli, Chem. Phys. Lett., 290,502 (1998).

38. M. Capitelli, M. Cacciatore, R. Celiberto, F. Esposito, C. Gorse, and A. Laricchiuta, Plasma Phys. Repts., 25, 5 (1999).

39. J. R. Hiskes, Appl. Phys. Lett., 69,755 (1996).

40. M. B. Hopkins, 1996.

41. C. B. Fleddermann, J. H. Beberman, and J. T. Verdeyen, Journal of Applied Physics, 58, 1344 (1985).

42. S. K. Srivastava, E. Krishnakumar, and A. D. d. A. e Souza, Int. J. Mass Spectrom. Ion Processes, 107, (1991).

43. A. A. Howling, J.-L. Dorier, and C. Hollenstein, Appl. Phys. Lett., 62, 1341 (1993).

44. A. A. Howling, L. Sansonnens, J.-L. Dorier, and C. Hollenstein, Journal of Applied Physics, 70, 2939 (1994).

45. A. Lloret, E. Bertran, J. L. Andujar, A. Canillas, and J. L. Morenza, Journal of Applied Physics, 69,632 (1991).

46. S. Samukawa, Appl. Phys. Lett., 68, 316 (1996).

47. A. Yokozawa, S. Samukawa, and H. Ohtake, Jpn. J. Appl. Phys., 35, 2433 (1996).

48. T. H. Ahn, K. Nakamura, and H. Sugai, Plasma Sources Sci. Technol., 5, 139 (1996).

49. T. Shibayama, H. Shindo, and Y. Horiike, Plasma Sources Sci. Technol., 5,254 (1996).

50. D. M. McCorkle, W. X. Ding, C. Y. Ma, and L. A. Pinnaduwage, J. Phys. D, 32, 46 (1999).

51. L. R. Martin and S. J. Harris, Appl. Phys. Lett., 59, 1911 (1991).

52. A. A. Fridman, L. Boufendi, T. I-Ibid, B. V.
Potapkin, and A. Bouchoule, Journal of Applied Physics, 79, 1303 (1996). 